\title{
LAS HACIENDAS LOCALES DE SEVILLA Y BARCELONA: UN ANÁLISIS DE CORRELACIÓN DE LA POLÍTICA PRESUPUESTARIA DURANTE EL PERÍODO 1986-1990.
}

Bartolomé PÉREZ RAMÍREZ *

Pablo LEÓN NOGALES *

\section{INTRODUCCIÓN.}

En este trabajo trataremos de hallar la correlación habida en las Políticas Fiscales (tanto en la vía de ingresos como de gastos públicos) de dos importantes Ayuntamientos españoles (Barcelona y Sevilla) durante el periodo presupuestario que va de 1986 a 1990, corporaciones con un rasgo común importante como han sido los acontecimientos de 1992 (la Expo en la ciudad de Sevilla y las Olimpiadas en la ciudad de Barcelona). A nuestro entender, y siempre desde una óptica estrictamente metodológica, los dos eventos de 1992 han sido un medio de hacer Política Económica, no un fin en si mismo. Por lo tanto, estos dos acontecimientos han de ser tratados como instrumentos para el crecimiento, modernización y desarrollo de las dos ciudades, con efectos positivos y muy beneficiosos para las economías barcelonesa y sevillana. Por otro lado, no podemos olvidar los dos aspectos que consideramos fundamentales:

1) El papel trascendental que han jugado las ciudades de Barcelona y Sevilla en variables económicas tales como Consumo Público e Inversiones Públicas (obras de infraestructura, viviendas y bienestar comunitario, deportes y juventud, etc.), que han alcanzado cifras realmente importantes, tanto por la vía de la oferta (cantidad y calidad de servicios prestados) como por la de demanda (creciente demanda social de servicios públicos),

\footnotetext{
*. Profesores del departamento de Económica Aplicada I de la Universidad de Sevilla.
} 
que mejoran, por una parte, el bienestar social de los ciudadanos, pero que requieren por otra parte la captación de recursos suficientes para afrontar dichos gastos.

2) El protagonismo que han tenido dos Corporaciones Locales como Barcelona y Sevilla en el ámbito económico, social, cultural, de ocio, etc. Las grandes obras de infraestructura viaria, los transportes y las comunicaciones, las urbanizaciones, las obras de acondicionamiento, construcción, rehabilitación y conservación de edificios, la creación de instalaciones deportivas, etc., son algunas de las cuantiosas inversiones en inmovilizado material que se han realizado. Todo ello ha constituido un importante elemento dinamizador de la economía de dichas ciudades, aunque por supuesto no el único.

Basándonos en los análisis más simples de dependencia entre las variables que vamos a estudiar, es decir, los análisis de tipo lineal, hemos obtenido una serie de correlaciones simples entre algunos de los capítulos del presupuesto de Ingresos Públicos, (Impuestos Directos, Impuestos Indirectos, Transferencias Corrientes y Variación de Pasivos Financieros), así como del Presupuesto de Gastos Públicos, (Gastos de Personal, Gastos en Bienes Corrientes y Servicios, Inversiones Reales y Variación de Pasivos Financieros), para Barcelona y Sevilla.

\section{ANÁLISIS DE CORRELACIÓN ENTRE LA POLÍTICA DE INGRESOS DE SEVILLA Y BARCELONA EN EL PERIODO 1986-1990}

En este apartado vamos analizar la correlación existente entre los cuatro capítulos de ingresos mencionados anteriormente. A continuación se detallan las variables usadas en el análisis:

ID_SEV Impuestos Directos. Presupuestos de Sevilla.

ID_BAR Impuestos Directos. Presupuestos de Barcelona.

II_SEV Impuestos Indirectos. Presupuestos de Sevilla.

II_BAR Impuestos Indirectos. Presupuestos de Barcelona.

TC_SEV Transferencias Corrientes. Presupuestos de Sevilla.

TC_BAR Transferencias Corrientes. Presupuestos de Barcelona.

PF_SEV Pasivos Financieros. Presupuestos de Sevilla.

PF_BAR Pasivos Financieros. Presupuestos de Barcelona. 


\section{II.1. Impuestos Directos:}

Desde 1986 a 1989 se contemplan dos artículos dentro de este Capítulo I el artículo de Renta, que recogía como conceptos más importantes en términos cuantitativos los impuestos municipales (Contribución Territorial Urbana Radicación y Licencias Fiscales), y los artículos de Capital (Impuestos sobre el Incremento de Valor de los Terrenos e Impuestos sobre solares). En e] ejercicio de 1990, con la entrada en vigor de la Ley 39/1988, de 28 de diciembre, reguladora de las Haciendas Locales, desaparece el artículo sobre Ic Renta, y continúan sólo los artículos sobre el CapitaI, pero con nuevas connotaciones, ya que se incorpora el Impuesto sobre Bienes Inmuebles (de naturaleza rústica y urbana), y el Impuesto sobre Vehículos de Tracción Mecánica, que antes era un impuesto indirecto.

La correlación obtenida entre los ingresos por impuestos indirectos pare Barcelona y Sevilla es muy alta en el período estudiado (ver cuadro I del Anexo) El valor del coeficiente de correlación, 0,9869 , denota que las dos Corporaciones Locales han optado por una presión fiscal potenciadora de la imposición directe en parecidos términos. En este caso se aprecian dos de las principales características intrínsecas de la imposición directa: Por un lado la progresividad, y por otrc la personalidad.

Por lo tanto, podemos afirmar que, a medida que han aumentado los ingresos por Impuestos Directos en el Ayuntamiento de Barcelona, también lo han hechc en el Ayuntamiento de Sevilla. Nota aparte, es el fuerte peso específico de los impuestos sobre la Renta en el período 1986-1989, y la potenciación de la tributación sobre el Capital (el Impuesto sobre Bienes Inmuebles "IBI") y la incorporación del Impuesto sobre Vehículos de Tracción Mecánica en 1990 er estas dos Corporaciones.

\section{II.2. Impuestos Indirectos:}

En los ejercicios presupuestarios de 1986, 1987, 1988 y 1989 se contemplar dos artículos en la composición del capítulo II. El que hacía referencia a] Consumo, que recogía como conceptos más importantes el Impuesto Municipa] sobre Gastos Suntuarios (apuestas en espectáculos públicos, cuotas de entrada er sociedades o círculos de recreo, etc.), y los Impuestos sobre Circulación de Vehículos y Publicidad. Desde enero de 1990 queda derogado el Impuestc Municipal sobre Gastos Suntuarios; el Impuesto sobre Circulación de vehículos que pasa a ser un impuesto directo y además con una nueva denominaciór "Impuesto sobre Vehículos de Tracción Mecánica; y aparece un nuevo impuesto 
el Impuesto sobre Construcciones, Instalaciones y Obras, que domina, si no íntegramente, sí con un porcentaje bastante alto la imposición indirecta.

La correlación que ha existido entre la imposición indirecta de Sevilla y Barcelona es baja en el período analizado, el coeficiente de correlación (ver cuadro l del Anexo) toma un valor de 0,5099, de modo que la relación es positiva, pero débil en esta parcela de la política impositiva de estas dos corporaciones. Ello es debido a que, mientras en los presupuestos de Barcelona el Capítulo II ha crecido de forma bastante irregular, en los de Sevilla si ha existido esa regularidad (que nada tiene que ver con grandes incrementos), salvo para 1990, ejercicio en el que el Impuesto sobre Circulación de Vehículos pasa a ser impuesto directo.

Observamos también cómo en el Ayuntamiento de Barcelona se ha aumentado la imposición fiscal directa a costa de disminuir la imposición indirecta, ya que la correlación entre ID_BAR e II_BAR es negativa y alta $(-0,8472)$, es decir, mientras que los ingresos por impuestos directos van creciendo, los ingresos por impuestos indirectos decrecen. En cambio, para Sevilla no ocurre lo mismo, ya que la correlación entre ID_SEV e II_SEV es casi nula $(-0,0939)$, lo que indica que los caminos seguidos por estos dos tipos de imposición han sido totalmente independientes el uno del otro.

\section{II.3. Transferencias Corrientes:}

El Capítulo de Transferencias Corrientes tiene su fundamento jurídico en el artículo 142 de la Constitución española: "Las Haciendas Locales deberán disponer de los medios suficientes para el desempeño de las funciones que la Ley atribuye a las Corporaciones respectivas y se nutrirán fundamentalmente de Tributos propios y de la participación en los del Estado y de las Comunidades Autónomas".

La política de ingresos tiene en esta vía un pilar básico que sustenta aproximadamente a la tercera parte de la misma, ya que las Transferencias Corrientes (que son entradas de recursos) vía participación en los tributos del Estado son de vital importancia para la financiación de la Política Económica de los mencionados Ayuntamientos.

La relación entre las Transferencias Corrientes de Barcelona y Sevilla es muy alta en el período analizado; el coeficiente de correlación obtenido es de 0,9443 , es decir, que existe una relación directa fuerte entre el Capítulo IV de los Municipios de Barcelona y Sevilla. Puesto que el mecanismo de participación de cualquier Municipio en los ingresos del Estado es automático, igual que el 
mecanismo que siguen las Comunidades Autónomas, parece lógico que esta correlación sea alta.

Entre otros baremos para obtener la participación de los Municipios en los tributos del Estado, nos encontramos con el Esfuerzo Fiscal, que se define comc la suma de los tres primeros capítulos de ingresos (Impuestos Directos, Impuestos Indirectos y Tasas y Otros Ingresos). La correlación entre estas dos magnitudes para Barcelona y Sevilla es bastante alta (ver cuadro III del Anexo), con ur coeficiente de correlación lineal de 0,9849, parece lógica la observación de que las Transferencias Corrientes de estas dos Corporaciones sigan una evoluciór. parecida.

\section{II.4. Variación de Pasivos Financieros:}

Dentro de este capítulo hay que tener en cuenta sobre todo el artículo denominado "Préstamos Recibidos a Largo Plazo" (recursos exclusivamente destinados a la financiación del Presupuesto de Inversiones, es decir, para financiar operaciones de capital), donde se asienta buena parte de la Política de Ingresos de los citados Ayuntamientos, puesto que el objetivo principal de este Capítulo es conseguir una Política de Inversiones fuerte. Es una de las piezas básicas en la obtención de recursos y, sin duda alguna, marcan una de las pautas más interesantes en la evolución de los Presupuestos de Ingresos de ambos Municipios. Hemos observado una línea ascendente en la evolución de este capítulo para ambas Corporaciones; por lo tanto, existe una política de progresivo endeudamiento a largo plazo por parte de las mismas, con una participación además cada vez mayor en relación al total de ingresos, de modo que se están reduciendo los porcentajes de ingresos por otros Capítulos, y se incrementan en gran medida los pasivos financieros. En definitiva, la conclusión es que se opta por el endeudamiento.

La correlación existente entre los Ingresos percibidos por Pasivos Financieros en los Ayuntamientos de Barcelona y Sevilla es muy alta en el quinquenio estudiado. El coeficiente de correlación asociado (ver cuadro IV del Anexo) vale 0,9648 , lo que nos muestra una relación fuerte entre el Capítulo IX de ambas Corporaciones. Y ello como consecuencia de una Política de endeudamiento creciente y paralela, es decir, a la vez que se incrementan los Pasivos Financieros en la Política Presupuestaria (vía ingresos) del Ayuntamiento de Sevilla, también lo hacen en los Presupuestos de Barcelona. 


\section{ANÁLISIS DE CORRELACIÓN ENTRE LA POLÍTICA DE GASTOS DE SEVILLA Y BARCELONA EN EL PERÍODO 1986-1990.}

Estudiaremos en esta parte la correlación existente entre los capítulos descritos en la introducción de este trabajo, para la Política de gastos. Antes de iniciar el análisis, vamos a definir las variables que usaremos:

GP_SEV Gastos de Personal. Presupuestos de Sevilla. GP_BAR Gastos de Personal. Presupuestos de Barcelona.

GB_SEV Gastos en Bienes Corrientes y Servicios. Presupuestos de Sevilla. GB_BAR Gastos en Bienes Corrientes y Servicios. Presupuestos de Barcelona. IR_SEV Inversiones reales. Presupuestos de Sevilla. IR_BAR Inversiones reales. Presupuestos de Barcelona. PF_SEV Pasivos Financieros. Presupuestos de Sevilla. PF_BAR Pasivos Financieros. Presupuestos de Barcelona.

En primer lugar, vamos a observar los Gastos de Personal y los Gastos en Bienes Corrientes y Servicios (ver cuadro V del Anexo).

\section{1. Gastos de Personal:}

Es el Capítulo I de la estructura presupuestaria vía Gasto Público, y está compuesto por las Remuneraciones de Personal (retribuciones básicas, otras remuneraciones, complementos farniliares, cuotas de la Seguridad social, cuotas de la MUNPAL, clases pasivas, etc.). Es uno de los grandes bloques donde se asienta la Política de Gastos en ambos Ayuntamientos. Sin embargo, en este periodo los gastos de personal van disminuyendo en relación al total del Presupuesto de Gastos, lo que en Política Económica significa la apuesta por una reducción del "Consumo Público" (ya que uno de sus componentes es este capítulo I de gastos).

La correlación entre los gastos de personal en los Ayuntamientos de Barcelona y Sevilla es alta durante el periodo analizado. El coeficiente de correlación lineal resultante es 0,8465 , lo que nos confirma una relación fuerte en este capítulo para ambos Municipios. No obstante, cabe resaltar que la relación no es muy alta (es decir, que en el presente trabajo han aparecido correlaciones más fuertes).

\section{III.2. Compras en Bienes Corrientes y Servicios:}

Es el capítulo II del Presupuesto de Gastos, y destaca en gran medida el artículo denominado "Gastos Especiales para el Funcionamiento de los servicios", que en el 
ejercicio de 1990 aparece con la titulación "Material, Suministros y otros". A est artículo corresponden los gastos de vestuario, material técnico y especial, combusti. bles, suministros de gas, agua, electricidad, teléfono, productos de limpieza, etc. Otrc artículo que destaca es el de Conservación, Mantenimiento y Reparación Ordinaria de Inversiones (de vehículos, de maquinaria e instalaciones, y de otras inversiones tale: como infraestructura y bienes naturales, edificios y otras construcciones, etc.).

Hay que tener muy en cuenta esta parcela del Presupuesto de gastos públicor locales, ya que las previsiones para el futuro indican que este capítulo es ur capítulo al alza, porque estamos observando una pequeña revolución en los gastos locales destinados al mantenimiento, conservación y reparación de las fuertes inversiones efectuadas en los últimos años de vida municipal. La Hacienda Loca altamente inversora de la última década nos conduce ahora a unas previsiones $d \epsilon$ gastos corrientes por este tipo de conceptos muy elevados, lo que va a implicar ur tratamiento especial al gasto corriente local en los próximos ejercicios presupuestarios, con un elevado riesgo de envejecimiento del capital fijo urbano, en caso de nc actuar en esta dirección. Por lo tanto, el gasto público local de los próximos años estará influido por una dinámica expansiva en la zona del gasto corriente, en ur trasvase de los gastos de inversión a gastos corrientes, es decir, a unos crecimientos en el capítulo II (sobre todo en Mantenimiento, Conservación y Reparación de Inversiones), y a unos estancamientos o descensos en el capítulo VI (Inversiones Reales), debido al amortiguamiento en lo que es inversión.

También es importante reseñar cómo se está produciendo una metarmorfosis en el concepto de gasto público de inversión, en el sentido de internalizar dentrc del presupuesto lo que todavía hoy es una inversión que se puede realizar o no en función de las posibilidades financieras de cada Ente Local. Hoy en día, y sirva como ejemplo, pavimentar o acerar una calle, mejorar el alumbrado público. mantener parques y jardines, etc., es considerado por los ciudadanos -contribuyentes al fin y al cabo- como un derecho, como algo de obligado cumplimiento. que hay que ejecutar con independencia del grado de financiación exógena de] presupuesto, y ello implica en términos presupuestarios aumentar los gastos en bienes corrientes y servicios a costa de eliminar gastos de inversión.

La correlación que existe en este capítulo para los Municipios de Barcelona y Sevilla es alta durante el quinquenio estudiado. El coeficiente de correlación que se obtiene es 0,9234 , según podemos ver en el cuadro $\mathrm{V}$ del Anexo, lo que demuestra que existe una relación directa fuerte dentro de este capítulo para ambas Corporaciones. Además, se observa una estrecha relación entre los dos componentes del consumo público (gastos de personal y gastos en bienes corrientes y servicios) en Barcelona y Sevilla, medidos por unos coeficientes de 
correlación de 0,9107 y 0,9794, respectivamente. Ello es lógico, ya que existe bastante relación entre los capítulos I y II.

\section{III.3. Inversiones Reales:}

Nos referimos al capítulo VI, capítulo de gran relevancia en el análisis cuantitativo, debido a la importancia de sus cifras dentro del montante total de gastos de los dos Ayuntamientos a que nos estamos refiriendo en este trabajo, y uno de los principales pilares donde se ha asentado la política de gastos a niveI municipal.

Las inversiones efectuadas por el Ayuntamiento de Sevilla con motivo de la celebración de la Exposición Universal de 1992 han sido importantes, aunque en los últimos años han bajado los porcentajes. Ello es debido, junto con otras causas, a un cierto amortiguamiento del gran esfuerzo inversor llevado a cabo en estas últimas legislaturas de Corporaciones Locales democráticas.

En este apartado puede observarse un hecho importante: En el Ayuntamiento de Barcelona existe una disminución en lo que a inversión fija se refiere, fruto entre otras cosas del enorme esfuerzo inversor llevado a cabo en la década de los 60 y parte de los 70, donde Barcelona se convirtió en uno de los principales focos receptores de emigrantes, y ello trajo consigo cambios, tanto cualitativos como cuantitativos, en la demanda de servicios públicos y de las infraestructuras y equipamientos urbanos (vivienda, urbanismo, cultura, transportes, bienestar comunitario, etc.). El fuerte crecimiento de Ia demanda tuvo una respuesta por el lado de la oferta municipal altamente positiva y diversificada, en el sentido de aumentar las infraestructuras, de modo que llegaron las inversiones en el urbanismo moderno: Mejoras en la red viaria, proliferación de barriadas periféricas, que surgieron a la sombra de la emigración y que supusieron fuertes inyecciones de capital en pavimentaciones de calles, alumbrado público, parques y jardines, colegios, etc. Es decir, el gran esfuerzo inversor desarrollado en las últimas décadas en Barcelona ha conducido a los responsables de esta Corporación en estos últimos años a practicar una Política Económica de cierta disminución o amortiguamiento de los gastos relacionados con el capítulo VI, ya que, en términos generales, posee unas buenas infraestructuras y un urbanismo marcado por tintes modernos y vanguardistas. Ahora, en cambio, se encuentran en una fase de conservación, reparación y mantenimiento de las fuertes inversiones realizadas en épocas anteriores, y ello implica en términos presupuestarios reducir gastos en el capítulo VI y aumentarlos en el capítulo II.

Atendiendo por tanto al comentario anterior, deberíamos concluir que entre la evolución de este capítulo en los Municipios de Sevilla y de Barcelona no 
debería existir relación alguna, es decir, cada ciudad debe tener una evolución independiente de la otra.

En el cuadro VI estudiamos la correlación que existe entre los gastos de inversión por un lado, y por otro lado la correlación en el capítulo de Variación de Pasivos Financieros (amortización de deuda pública y amortización de los préstamos recibidos a medio y largo plazo). En cuanto a los gastos de inversión. se corrobora lo anterior. El coeficiente de correlación entre Barcelona y Sevilla en cuanto a estos gastos es 0,1970 , lo que muestra una relación muy pequeña (la más baja de las estudiadas hasta ahora).

\section{III.4. Variación de Pasivos Financieros:}

Este capítulo está dominado por el artículo de Amortización de préstamos a medio y largo plazo. Lo más destacable es que la carga financiera va creciendo con el paso de los años en ambos Municipios, como consecuencia de los incrementos producidos tanto en el capítulo III (Gastos Financieros) como en el capítulo IX (Variación de Pasivos Financieros) de la Política de gastos municipal en relación a los ingresos corrientes, que se traduce en términos económicos por la opción de un endeudamiento progresivo, es decir, al incrementar los ingresos por pasivos financieros (préstamos recibidos) en la política de ingresos, se incrementa la amortización por dichos préstamos recibidos o por la deuda pública emitida. Por lo tanto, y como conclusión, existe un aumento del capítulo IX de gastos.

Al igual que ocurre con el capítulo IX de ingresos, la correlación para este capítulo en el presupuesto de gastos de Barcelona y Sevilla es muy alta, medida por el coeficiente de correlación en 0,9863 . Por tanto, existe una fuerte relación positiva entre ambas políticas de endeudamiento, y como hemos comentado antes, se traduce en unas políticas paralelas de endeudamiento creciente.

\section{CONCLUSIONES}

Como punto final de este análisis, destacamos entre los aspectos más sobresalientes, los siguientes:

1. La aplicación de una política fiscal potenciadora de la imposición directa, sobre todo por la vía de los impuestos sobre la renta en los primeros cuatro ejercicios del período analizado, y en el último, al cambiar la estructura presupuestaria, se potencia la tributación sobre el capital (con e] 
Impuesto sobre Bienes Inmuebles "IBI"). Además, la correlación existente es muy alta, con un coeficiente de corrrelación de 0,9869.

2. La imposicion indirecta es pequeña, y la correlación entre los ingresos por impuestos indirectos de Barcelona y Sevilla no es muy alta en el período analizado; el coeficiente de correlación calculado es de 0,5099, lo que indica una correlación débil; aun así, en todo caso positiva.

3. La política de ingresos tiene un pilar importante en las transferencias corrientes (entrada de recursos vía participación en los tributos del Estado), ya que suponen aproximadamente Ia tercera parte de estos ingresos. La correlación de esta capítulo para los dos Municipios es también alta y positiva, con un coeficiente de correlación de 0,9443.

4. El capítulo IX del presupuesto de ingresos (y sobre todo el artículo de Préstamos Recibidos a Largo Plazo) que hay que tener muy en cuenta a la hora de realizar el análisis cuantitativo de los presupuestos de estos municipios, ya que hemos observado una línea ascendente en la participación de este capítulo en el total de ingresos para ambas ciudades, decrementándose la participación de otros ingresos. La correlación de este capítulo en ambas ciudades es bastante a!ta, medida por el coeficiente de correlación en 0,9648 .

5. De Ia política de gastos destacamos, por una parte, la importancia del capítulo II (gastos en bienes corrientes y servicios), donde se alcanzan cifras muy importantes, aunque mayores en Barcelona que en Sevilla. Este hecho implica efectivamente un tratamiento muy especial a los gastos corrientes por parte de la Corporación de Barcelona, para no caer en el envejecimiento de capital fijo urbano, y ello ha conducido a una cierta dinámica expansiva en la vertiente del gasto corriente a costa de disminuir los gastos de inversiones. La correlación entre el capítulo II de Barcelona y Sevilla es alta durante el período analizado, medida por el coeficiente de correlación en 0,9234 .

6. En el capítulo VI, de Inversiones Reales, es relevante el análisis cuantitativo por el valor de sus cifras, aunque en términos de distribución porcentual está por encima Sevilla; así, durante los primeros años de la investigación, (1986, 1987 y 1988), se alcanzan en Sevilla cifras de inversión realmente significativas, y en los dos últimos ejercicios (1989 y 1990) 
bajan los porcentajes y se produce un cierto amortiguamiento, aunque nc obstante el esfuerzo inversor desarrollado ha sido importante. Sin embargo. en Barcelona se observa una disminución en la inversión fija, debido entre otras cosas al gran esfuerzo inversor de los años 60 y 70 . En la actualidad. se encuentra en fase de mantenimiento y reparación de las inversiones realizadas en épocas anteriores.

Todo ello, nos lleva a concluir una característica importante en el análisis de correlación en las inversiones reales entre Sevilla y Barcelona, y es la poca significatividad de la misma. El coeficiente de correlación que se obtiene es de 0,0917 , que nos conduce a deducir que existe una relación significativamente poco importante en la evolución del capítulo VI para estas dos ciudades.

7. Por último, diremos que la carga financiera va creciendo con el paso de los años en los dos Municipios, como consecuencia de las variaciones positivas en los gastos financieros (capítulo III de gastos) y en las variaciones de pasivos financieros (capítulo IX de gastos), por la amortización, tanto del capital como de los intereses de los préstamos a medio y largo plazo, o por la deuda pública emitida. La correlación es bastante alta, con un coeficiente de correlación de 0,9863 .

\section{BIBLIOGRAFÍA.}

AYUNTAMIENTO DE BARCELONA (Servicio de Estadística) (1986, 1987, 1988, 1989 y 1990): Anuarios estadísticos de la ciudad de Barcelona. Años 1986, 1987, 1988, 1989 y 1990. Barcelona. AYUNTAMIENTO DE BARCELONA (Gaceta Municipal) (1986, 1987, 1988, 1989 y 1990):

Presupuestos municipales. Años 1986 (I), (II), (III), (IV) y (V), 1987 (I), (II) y (III), 1988 (I), (II) y (III), 1989 (I) y (II), y 1990. Barcelona.

AYUNTAMIENTO DE SEVILLA (1987, 1988, 1989, 1990 y 1991): Anuarios estadísticos de la ciudad de Sevilla. Años 1986 (Tomos I y II), 1987, 1988, 1989 y 1990. Delegación Municipal de Estadística. Sevilla.

AYUNTAMIENTO DE SEVILLA (1986, 1987, 1988, 1989 y 1990): El Presupuesto municipal, ejercicios de 1986, 1987, 1988, 1989 y 1990 . Sevilla.

CARRILLO BENITO, E. (1985): La Hacienda Local en la Ley 7/85. Ponencia presentada en la Delegación Interprovincial del I.E.A.L.. Sevilla.

CASTELLS OLIVERES, A. (1988): Hacienda Autonómica. Una perspectiva del federalismo fiscal. Ariel. Barcelona.

CASTELLS OLIVERES, A. (1984): Financiación y crisis fiscal de las grandes ciudades. Centro de Estudios de Planificación (CEP). Barcelona.

FUENTES QUINTANA, E. (1986): Introducción y presupuesto. Hacienda Pública Española. Madrid. 
MINISTERIO DE ECONOMIA Y HACIENDA (Secretaría de Estado de Hacienda) (1989): Evolución de la financiación de las Corporaciones Locales en el periodo 1986-1989. Dirección General de Coordinación con las Haciendas Territoriales. Madrid.

PEREZ RAMIREZ, B. (1991): El sector público en los últimos cinco años (1986-1990): Especial referencia a los Municipios de Barcelona, Huelva y Sevilla. V Reunión ASEPELT-ESPAÑA (edita la Caja de Canarias). Gran Canaria.

VALLES FERRER, J. (1985): La financiación de las Haciendas Locales en España hoy. Conferencia dictada en la IV Semana de Estudios sobre Economía de las Corporaciones Locales, organizada por el Centro de Estudios Municipales y de Cooperación Interprovincial de las Diputaciones de Almería, Granada, Jaén y Málaga. I.E.A.L. Granada.

VALLES FERRER, J. (1985): Los presupuestos de las Corporaciones Locales ante la nueva Ley de Bases de Régimen Local (los problemas de unificación, coordinación, valoración y saneamiento). Conferencia dictada en las Jomadas de Estudios sobre Presupuesto y Contabilidad de las Entidades Locales, organizada por la Delegación Interprovincial del I.E.A.L. Sevilla.

VALLES FERRER, J. (1991): La Hacienda Local Postconstitucional (Principios, evolución y realidad) (1978-1990). Edita El Monte Caja de Huelva y Sevilla. Sevilla.

\section{ANEXO}

Correlacion entre las variables de ingresos y gastos Estudiadas en el trabajo.

CUADRO I

\begin{tabular}{lcccc}
\hline \multicolumn{5}{c}{ Correlaciones del tipo r(x,y) } \\
\hline & ID_SEV & II_SEV & ID_BAR & II_BAR \\
\hline ID_SEV & 1 & $-0,0939$ & 0,9869 & - \\
II_SEV & $-0,0939$ & 1 & - & 0,5099 \\
ID_BAR & 0,9869 & - & 1 & $-0,8472$ \\
II_BAR & - & 0,5099 & $-0,8472$ & 1 \\
\hline
\end{tabular}

Fuente: Elaboración propia

CUADRO II

\begin{tabular}{ccc}
\hline \multicolumn{3}{c}{ Correlaciones deI tipo $\mathrm{r}(\mathrm{x}, \mathrm{y})$} \\
\hline & TC_SEV & TC_BAR \\
\hline TC_SEV & 1 & 0,9443 \\
TC_BAR & 0,9443 & 1 \\
\hline
\end{tabular}

Fuente: Elaboración propia. 
CUADRO III

\begin{tabular}{lcc}
\hline \multicolumn{3}{c}{ Correlaciones del tipo $\mathrm{r}(\mathrm{x}, \mathrm{y})$} \\
\hline & EF_SEV & EF_BAR \\
\hline EF_SEV & 1 & 0,9849 \\
EF_BAR & 0,9849 & 1 \\
\hline
\end{tabular}

Fuente: Elaboración propia

CUADRO IV

\begin{tabular}{lcc}
\hline \multicolumn{3}{c}{ Correlaciones del tipo $\mathrm{r}(\mathrm{x}, \mathrm{y})$} \\
\hline & PF_SEV & PF_BAR \\
\hline PF_SEV & 1 & 0,9648 \\
PF_BAR & 0,9648 & 1 \\
\hline
\end{tabular}

Fuente: Elaboración propia

CUADRO V

\begin{tabular}{lcccc}
\hline \multicolumn{5}{c}{ Correlaciones del tipo r(x,y) } \\
\hline & GP_SEV & GB_SEV & GP_BAR & GB_BAR \\
\hline GP_SEV & 1 & 0,9794 & 0,8465 & - \\
GB_SEV & $-0,9794$ & 1 & - & 0,9234 \\
GP_BAR & 0,8465 & - & 1 & 0,9107 \\
GB_BAR & - & 0,9234 & 0,9107 & 1 \\
\hline
\end{tabular}

Fuente: Elaboración propia

CUADRO VI

\begin{tabular}{lcccc}
\hline \multicolumn{5}{c}{ Correlaciones del tipo r(x,y) } \\
\hline & IR_SEV & PF_SEV & IR_BAR & PF_BAR \\
\hline IR_SEV & 1 & - & 0,1970 & - \\
PF_SEV & - & 1 & - & 0,9863 \\
IR_BAR & 0,1970 & - & 1 & - \\
PF_BAR & - & 0,9863 & - & 1 \\
\hline
\end{tabular}

Fuente: Elaboración propia 


\section{Cuadro VIII}

EVOLUCIÓN DE LOS PRESUPUESTOS DE INGRESOS PER CÁPITA DEL AYUNTAMIENTO DE BARCELONA (1986-1990) (Pesetas/Habitantes)

\begin{tabular}{|c|c|c|c|c|c|c|c|c|c|c|c|}
\hline Cap. & Descripción & 1986 & $\%$ & 1987 & $\%$ & 1988 & $\%$ & 1989 & $\%$ & 1990 & $\%$ \\
\hline & Impuestos Directos & $17.509,6$ & 31,68 & $19.387,3$ & 32,34 & $22.840,1$ & 31,36 & $23.516,2$ & 30,69 & $32.402,3$ & 36,30 \\
\hline & Impuestos Indirectos & $2.562,0$ & 4,64 & $2.327,8$ & 3,88 & $2.388,7$ & 3,28 & $2.695,1$ & 3,52 & $1.259,3$ & 1,41 \\
\hline & Tasas y otros ingresos & $10.227,4$ & 18,50 & $10.208,7$ & 17,03 & $11.495,9$ & 15,78 & $11.674,0$ & 15,23 & $9.768,1$ & 10,94 \\
\hline IV & Transferencias Corrientes & $17.831,6$ & 32,26 & $21.381,7$ & 35,67 & $25.054,9$ & 34,40 & $26.868,3$ & 35,06 & $28.446,9$ & 31,87 \\
\hline$V$ & Ingresos Patrimoniales & $1.386,1$ & 2,51 & $1.720,9$ & 2,87 & $1.905,7$ & 2,62 & $1.691,2$ & 2,21 & $3.577,0$ & 4,01 \\
\hline$A$ & $\begin{array}{l}\text { SUBTOTAL DE } \\
\text { OPERACIONES CORRIENTES }\end{array}$ & $49.516,7$ & 89,59 & $55.026,4$ & 91,80 & $63.685,3$ & 87,45 & $66.444,8$ & 86,70 & $75.453,6$ & 84,53 \\
\hline VI & Enajenación de Inversiones reales & 176,4 & 0,32 & 140,9 & 0,24 & 697,1 & 0,96 & 511,0 & 0,67 & 292,9 & 0,33 \\
\hline VII & Transferencias de Capital & 502,2 & 0,91 & - & - & - & - & 817,6 & 1,07 & 439,3 & - \\
\hline & $\begin{array}{l}\text { SUBTOTAL DE } \\
\text { OPERACIONES DE CAPITAL }\end{array}$ & 678,6 & 1,23 & 140,9 & 0,24 & 697,1 & 0,96 & $1.328,6$ & 1,73 & 732,2 & 0,33 \\
\hline & $\begin{array}{l}\text { SUBTOTAL DE } \\
\text { OPERACIONES NO FINANCIERAS }\end{array}$ & $50.195,3$ & 90,81 & $55.167,3$ & 92,04 & $64.382,4$ & 88,40 & $67.773,4$ & 88,43 & $76.185,8$ & 84,85 \\
\hline VIII & Variación Activos Financieros & 76,4 & 0,14 & 76,3 & 0,13 & 192,5 & 0,26 & 104,0 & 0,14 & 122,4 & 0,14 \\
\hline $\mathrm{X}$ & Variación Pasivos Financieros & $5.000,6$ & 9,05 & $4.695,5$ & 7,83 & $8.253,8$ & 11,33 & $8.759,9$ & 11,43 & $12.958,6$ & 14,52 \\
\hline & $\begin{array}{l}\text { SUBTOTAL DE } \\
\text { OPERACIONES FINANCIERAS }\end{array}$ & $5.077,0$ & 9,19 & $4.771,8$ & 7,96 & $8.446,3$ & 11,60 & $8.863,9$ & 11,57 & $13.081,0$ & 14,65 \\
\hline & TOTAL & $55.272,3$ & 100,00 & $59.939,1$ & 100,00 & $72.828,7$ & 100,00 & $76.637,3$ & 100,00 & $89.266,8$ & 100,00 \\
\hline
\end{tabular}

Fuente: Presupuesto Municipal. Ejercicios 1986-1990. Excmo. Ayuntamiento de Sevilla. Elaboración propia 


\section{Cuadro IX}

EVOLUCIÓN DE LOS PRESUPUESTOS DE GASTOS PER CÁPITA DEL AYUNTAMIENTO DE SEVILLA (1986-1990) (Pesetas/habitantes)

\begin{tabular}{|c|c|c|c|c|c|c|c|c|c|c|c|}
\hline Cap. & Descripción & 1986 & $\%$ & 1987 & $\%$ & 1988 & $\%$ & 1989 & $\%$ & 1990 & $\%$ \\
\hline & Gastos de personal & $13.322,3$ & 48,00 & $12.055,5$ & 37,89 & $14.441,0$ & 34,12 & $15.669,4$ & 28,10 & $17.923,0$ & 28,53 \\
\hline II $\cdot$ & Gastos en bienes corrientes y servicios & $3.868,1$ & 13,94 & $3.740,1$ & 11,76 & $4.313,0$ & 10,19 & $4.888,9$ & 8,77 & $6.087,3$ & 9,69 \\
\hline III & Gastos financieros & $2.070,8$ & 7,46 & $2.447,7$ & 7,69 & $3.514,1$ & 8,30 & $4.764,7$ & 8,54 & $6.552,9$ & 10,43 \\
\hline \multirow{3}{*}{$\begin{array}{l}\text { IV } \\
\text { A }\end{array}$} & Transferencias Corrientes & $1.899,5$ & 6,84 & $4.829,1$ & 15,18 & $5.713,4$ & 13,50 & $7.731,9$ & 13,87 & $9.825,7$ & 15,64 \\
\hline & SUBTOTAL DE & & & & & & & & & & \\
\hline & OPERACIONES CORRIENTES & $21.160,7$ & 76,24 & $23.072,4$ & 72,52 & $27.981,5$ & 66,11 & $33.054,9$ & 59,28 & $40.388,9$ & 64,28 \\
\hline VI & Inversiones Reales & $5.353,7$ & 19,29 & $7.123,4$ & 22,39 & $12.540,5$ & 29,63 & $12.641,5$ & 22,67 & $13.074,3$ & 20,81 \\
\hline VII & Transferencias de Capital & - & - & - & - & - & - & $7.203,4$ & 12,92 & $5.775,6$ & 9,19 \\
\hline \multirow[t]{2}{*}{ B } & $\begin{array}{l}\text { SUBTOTAL DE } \\
\text { OPERACIONES DE CAPITAL }\end{array}$ & $\mathbf{5 . 3 5 3 , 7}$ & 19,29 & $7.123,4$ & 22,39 & $12.540,5$ & 29,63 & $19.844,9$ & 35,59 & $18.849,9$ & 30,00 \\
\hline & $\begin{array}{l}\text { SUBTOTAL DE } \\
\text { OPERACIONES NO FINANCIERAS }\end{array}$ & $26.514,4$ & 95,53 & $30.195,8$ & 94,91 & $40.522,0$ & 95,74 & $52.899,8$ & 94,87 & $59.238,8$ & 94,28 \\
\hline VIII & Variación Activos Financieros & - & - & - & - & - & - & - & - & - & - \\
\hline IX & Variación Pasivos Financieros & $1.241,0$ & 4,47 & $1.620,6$ & 5,09 & $1.801,6$ & 4,26 & $2.861,8$ & 5,13 & $3.591,2$ & 5,72 \\
\hline \multirow[t]{3}{*}{$\mathrm{D}$} & SUBTOTAL DE & & & & & & & & & & \\
\hline & OPERACIONES FINANCIERAS & $1.241,0$ & 4,47 & $1.620,6$ & 5,09 & $1.801,6$ & 4,26 & $2.861,8$ & 5,13 & $3.591,2$ & 5,72 \\
\hline & TOTAL & $27.755,4$ & 100,00 & $31.816,4$ & 100,00 & $42.323,6$ & 100,00 & $55.761,6$ & 100,00 & $62.830,0$ & 100,00 \\
\hline
\end{tabular}

Fuente: Presupuesto Municipal. Ejercicios 1986-1990. Excmo. Ayuntamiento de Sevilla. Elaboración propia 


\section{Cuadro X}

EVOLUCIÓN DE LOS PRESUPUESTOS DE GASTOS PER CÁPITA DEL AYUNTAMIENTO DE BARCELONA (1986-1990) (Pesetas/habitantes)

\begin{tabular}{|c|c|c|c|c|c|c|c|c|c|c|c|}
\hline Cap. & Descripción & 1986 & $\%$ & 1987 & $\%$ & 1988 & $\%$ & 1989 & $\%$ & 1990 & $\%$ \\
\hline & Gastos de personal & $20.429,1$ & 36,96 & $21.951,1$ & 36,62 & $21.782,8$ & 29,91 & $22.964,9$ & 29,97 & $24.894,4$ & 27,89 \\
\hline & Gastos en bienes corrientes y servicios & $12.664,3$ & 22,91 & $14.054,8$ & 23,45 & $17.416,5$ & 23,91 & $18.475,3$ & 24,11 & $20.896,1$ & 23,41 \\
\hline II & Gastos financieros & $7.625,6$ & 13,80 & $8.676,7$ & 14,48 & $10.000,1$ & 13,73 & $10.437,8$ & 13,62 & $13.916,5$ & 15,59 \\
\hline V & Transferencias Corrientes & $4.353,2$ & 7,88 & $7.010,4$ & 11,70 & $7.893,7$ & 10,84 & $9.974,4$ & 13,02 & $11.354,7$ & 12,72 \\
\hline 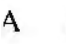 & \multicolumn{11}{|l|}{ SUBTOTAL DE } \\
\hline & OPERACIONES CORRIENTES & $45.072,2$ & 81,55 & $51.693,0$ & 86,24 & $57.093,1$ & 78,39 & $61.852,4$ & 80,71 & $71.061,7$ & 79,61 \\
\hline VI & Inversiones Reales & $8.484,0$ & 15,35 & $6.040,8$ & 10,08 & $10.657,1$ & 14,63 & $8.497,1$ & 11,09 & $6.069,4$ & 6,80 \\
\hline VII & Transferencias de Capital & 492,1 & 0,89 & $1.086,0$ & 1,81 & $3.447,4$ & 4,73 & $3.481,8$ & 4,54 & $7.840,6$ & 8,78 \\
\hline \multirow[t]{4}{*}{ B } & SUBTOTAL DE & & & & & & & & & & \\
\hline & OPERACIONES DE CAPITAL & $8.976,1$ & 16,24 & $7.126,8$ & 11,89 & $14.104,5$ & 19,37 & $11.987,9$ & 15,63 & $13.910,0$ & 15,58 \\
\hline & SUBTOTAL DE & & & & & & & & & & \\
\hline & OPERACIONES NO FINANCIERAS & $54.048,3$ & 97,79 & $58.819,8$ & 98,13 & $71.197,6$ & 97,76 & $73.831,3$ & 96,34 & $84.971,7$ & 95,19 \\
\hline VIII & Variación Activos Financieros & 323,2 & 0,58 & 164,3 & 0,27 & 192,5 & 0,26 & 104,2 & 0,14 & 122,4 & 0,14 \\
\hline$X$ & Variación Pasivos Financieros & 900,6 & 1,63 & 955,1 & 1,59 & $1.438,5$ & 1,98 & $2.702,1$ & 3,53 & $4.172,7$ & 4,67 \\
\hline \multirow[t]{3}{*}{ 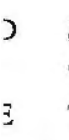 } & SUBTOTAL DE & & & & & & & & & & \\
\hline & OPERACIONES FINANCIERAS & $1.223,8$ & 2,21 & $1.119,4$ & 1,87 & $1.631,0$ & 2,24 & $2.806,3$ & 3,66 & $4.295,1$ & 4,81 \\
\hline & TOTAL & $55.272,1$ & 100,00 & $59.939,2$ & 100,00 & $72.828,6$ & 100,00 & $76.637,6$ & 100,00 & $89.266,8$ & 100,00 \\
\hline
\end{tabular}

"uente: Presupuesto Municipal. Ejercicios 1986-1990. Excmo. Ayuntamiento de Sevilla. Elaboración propia 


\section{Gráfico I}

EVOLUCION DE LOS GASTOS DE PERSONAL Y B. y S. PARA SEVILLA Y BARCELONA

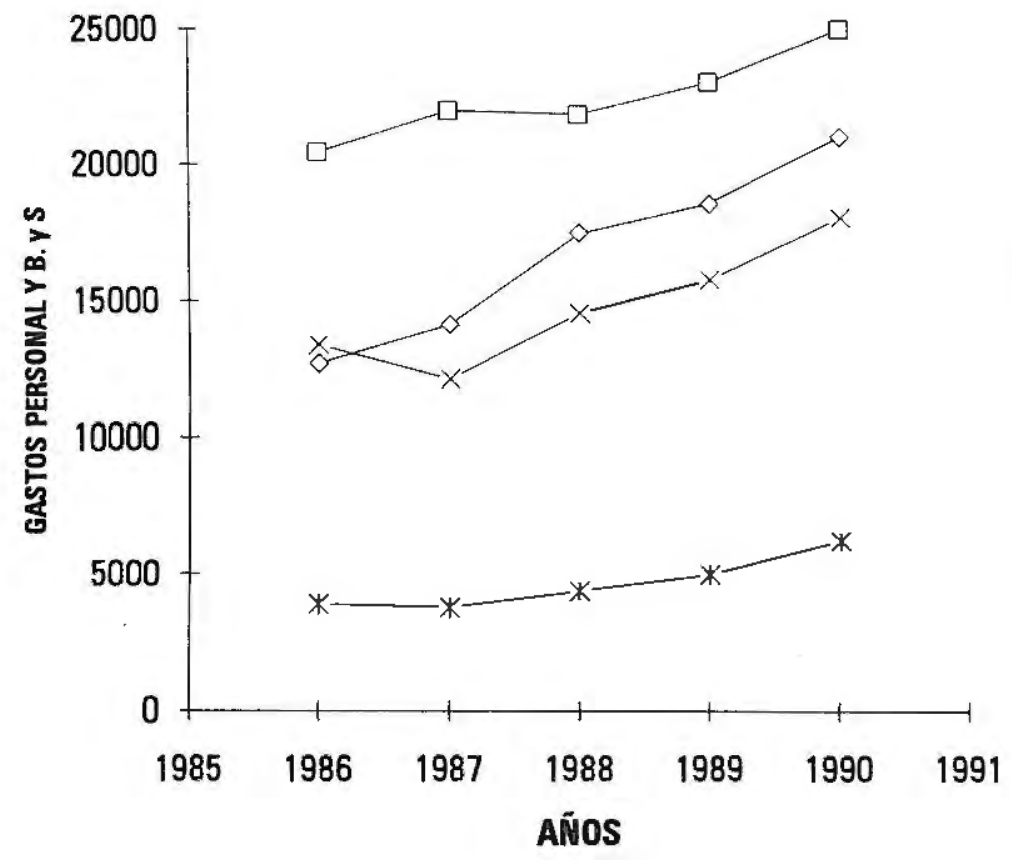

$\longrightarrow \times-$ Gastos Pers. Sevilla

——— Gastos B. y S. Sevilla

- Gastos Pers. Barcelona

$\sim$ Gastos B. y S. Barcelona 
Gráfico II

ESFUERZO FISCAL PER CAPITA PARA SEVILLA Y BARCELONA

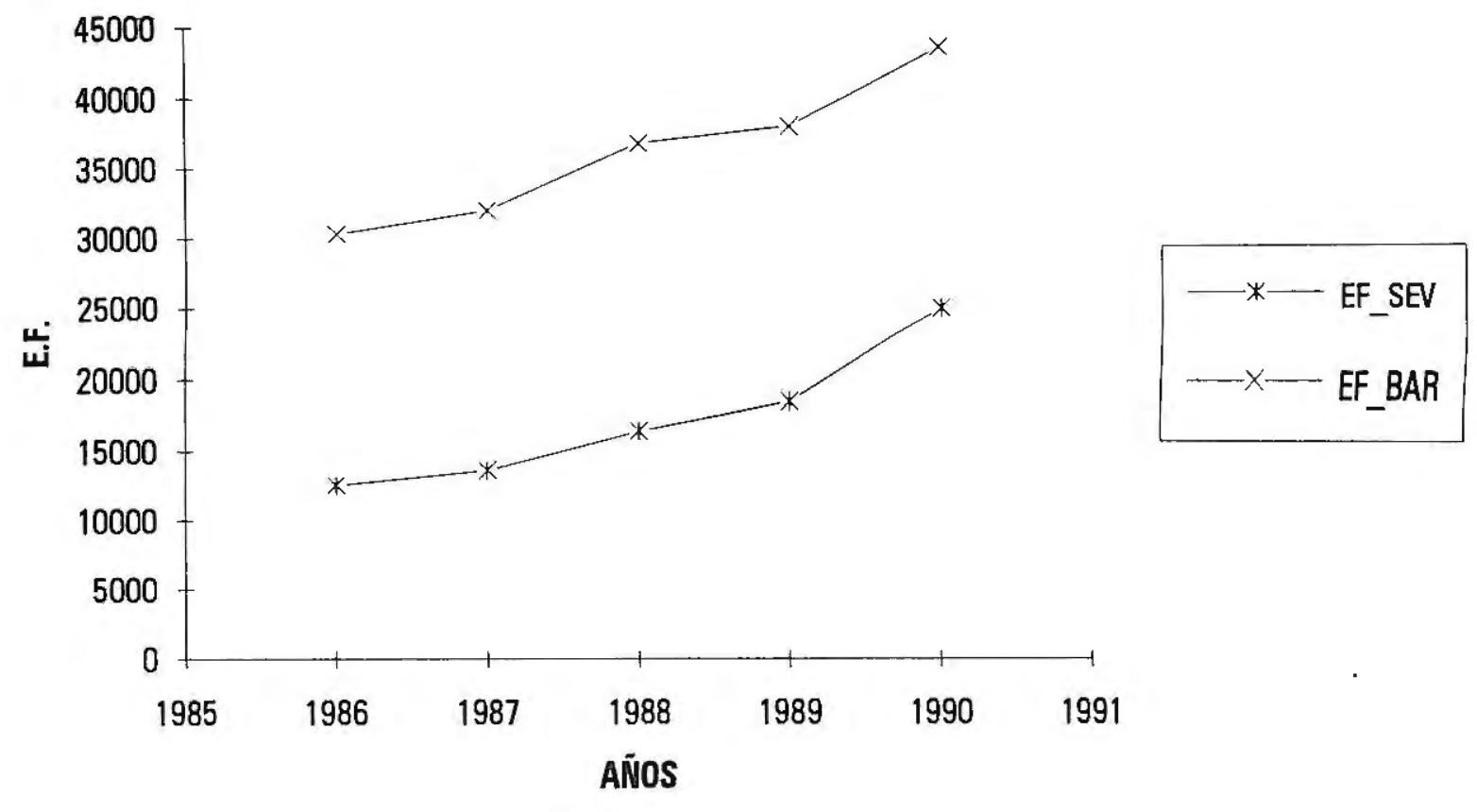

